\title{
How shall we judge a PET flow model?
}

\author{
Nils P. Johnson, MD, MS, and K. Lance Gould, $M^{a}{ }^{a}$ \\ a Weatherhead PET Center, Division of Cardiology, Department of Medicine, McGovern Medical \\ School at UTHealth and Memorial Hermann Hospital, Houston, TX
}

Received Aug 26, 2021; accepted Aug 26, 2021

doi: $10.1007 /$ s12350-021-02805-5

\section{See related article, pp. 2543-2550}

Starting over 70 years ago, ${ }^{1}$ clinicians began quantifying myocardial blood flow to diagnose and guide treatment. During the past seven decades, a large number of tools has been developed, both invasive and non-invasive. The ongoing utility of myocardial blood flow assessment testifies the underlying clinical need. Why does this patient have chest pain or dyspnea? Should I perform coronary angiography or how should I interpret its anatomy? Will the patient benefit from revascularization? And, if yes, which vessels require a procedure?

The ideal tool for studying myocardial blood flow possesses the following features. First, it should be noninvasive to avoid the small, but non-zero, risk of complications inherent to any catheterization and therefore facilitate serial assessment and require small or no radiation exposure. Second, minimal contraindications allow its application to the broadest range of patients regardless of body habitus, heart rate and rhythm, implanted devices, coagulopathy, and renal function. Third, the entire left ventricle must be evaluated independent of the complexity of coronary anatomy, including total occlusions, bypass grafts, and tortuous or calcified vessels. Fourth, since absolute flow depends on the amount of supplied muscle, only normalizing to myocardial mass (= perfusion) permits comparisons among patients, although serial exams in the same patient generally do not require this adjustment. Fifth,

Reprint requests: Nils P. Johnson, MD, MS, Weatherhead PET Center, Division of Cardiology, Department of Medicine, McGovern Medical School at UTHealth and Memorial Hermann Hospital, 6431 Fannin St., Room MSB 4.256, Houston, TX; Nils.Johnson@ uth.tmc.edu

J Nucl Cardiol 2022;29:2551-4.

$1071-3581 / \$ 34.00$

Copyright (C) 2021 American Society of Nuclear Cardiology. the technique should be repeatable with a known and acceptable test/retest variation under stable conditions. Finally, the resolution of the tool must permit distinction among the "revascularizable", vessels (generally at least 2 to $2.5 \mathrm{~mm}$ in reference diameter) and quantify their distribution territory as a fraction of the total left ventricle.

Since its initial description in animals ${ }^{2}$ and humans ${ }^{3}$ roughly 40 years ago for myocardial imaging, cardiac positron emission tomography (PET) has matured into the reference standard since it uniquely fulfills all of these ideal features. However, several software packages exist in current practice and, as admitted by a recent national statement on PET blood flow quantification, "significant variation remains among some vendor programs.", The current manuscript by Nesterov et al in this issue ${ }^{5}$ therefore provides a timely opportunity to consider how to judge existing PET flow software.

\section{SCORECARD FOR FLOW MODELS}

Table 1 summarizes our criteria for a scientifically valid and clinically practical flow model. First, the model should have been experimentally validated in animals using a reference standard such as an external flow probe ${ }^{6}$ or microspheres. Second, the model must be tested along the entire spectrum of flow that exists in nature, from the high levels in normal volunteers ${ }^{7}$ (distinct from clinical patients with mild risk factors that reduce average flows) to the low levels during ischemia ${ }^{8}$ (stress-induced perfusion defect with severe angina and/ or significant electrocardiographic changes). Note that flow levels for ischemia must be quantified on a regional basis - not globally - given its generally circumscribed pathophysiology. Obviously a flow model should work with all the major pharmacologic stress agents, including dipyridamole, ${ }^{9}$ adenosine, ${ }^{10}$ and regadenoson. ${ }^{11}$

Essentially every test in medicine displays some imprecision due to a combination of the physical limits of the technology plus biology itself. The net effect for myocardial imaging implies test/retest variation, even if 
Table 1. Scorecard for judging a PET flow package

\begin{tabular}{|c|c|c|c|}
\hline Feature & Description & Citation & $\mathbf{N}$ \\
\hline $\begin{array}{l}\text { Animal } \\
\text { validation }\end{array}$ & Comparison of PET against flow meter or microspheres & Yoshida $(1996)^{6}$ & 24 \\
\hline \multicolumn{4}{|l|}{ Flow spectrum } \\
\hline $\begin{array}{l}\text { Normal } \\
\text { volunteers }\end{array}$ & Rest and stress flow in young, healthy people (not patients) & Sdringola $(2011)^{7}$ & 125 \\
\hline Ischemia & $\begin{array}{l}\text { Stress-induced perfusion defect with severe angina or ECG } \\
\text { changes }\end{array}$ & Johnson $(2011)^{8}$ & 1674 \\
\hline Stress agents & $\begin{array}{l}\text { Interplay of vasodilator pharmacodynamics and radiotracer } \\
\text { timing }\end{array}$ & & \\
\hline Dipyridamole & & $\begin{array}{l}\text { Kitkungvan } \\
(2017)^{9}\end{array}$ & 120 \\
\hline Adenosine & & $\begin{array}{l}\text { Kitkungvan } \\
(2017)^{10}\end{array}$ & 127 \\
\hline Regadenoson & & Johnson $(2015)^{11}$ & 176 \\
\hline Test/retest & Separate biologic variation from stochastic effects of imaging & $\begin{array}{l}\text { Kitkungvan } \\
(2017)^{9}\end{array}$ & 708 \\
\hline Arterial input & $\begin{array}{l}\text { Scan-specific customization of arterial input to avoid } \\
\text { underestimation }\end{array}$ & Vasquez $(2013)^{12}$ & 288 \\
\hline $\begin{array}{l}\text { Clinical } \\
\text { outcomes }\end{array}$ & $\begin{array}{l}\text { Size/severity association with outcomes as modified by } \\
\text { treatment }\end{array}$ & Gould $(2021)^{14}$ & 5274 \\
\hline $\begin{array}{l}\text { Cost } \\
\text { effectiveness }\end{array}$ & Cost simulation ideally based on data from a randomized trial & Delgado $(2014)^{15}$ & $\mathrm{~N} / \mathrm{A}$ \\
\hline Randomized trial & Impact of PET-guided management on clinical outcomes & $\begin{array}{c}\text { Kitkungvan } \\
(2021)^{16}\end{array}$ & 1028 \\
\hline
\end{tabular}

done twice on the same day or a week or two apart under clinically stable circumstances. Short-term variation, as on the same day, largely reflects stochastic effects of the radiotracer and imaging chain amplified by the flow model, whereas long-term variation over a week or two also includes biologic fluctuations. We have demonstrated a $10 \%$ coefficient of variation in the short term that grows to $21 \%$ in the long term for rubidium- 82 and a retention model. ${ }^{9}$

The arterial input represents a vital yet often underappreciated component of flow quantification. While radiotracer concentration in the arterial circulation does not vary much spatially due to mixing during transit, both cardiac $\mathrm{PET}^{12}$ and magnetic resonance ${ }^{13}$ perfusion imaging display heterogeneity among potential sampling sites (left atrium, left ventricular cavity, ascending aorta, descending aorta). Reasons for this variation in PET include partial volume effects, spillover from adjacent structures, and motion of intrathoracic anatomy during cardiac and respiratory cycles as well as from gravity during supine positioning, imaging anisotropy, and co-registration of emission and transmission images that focuses on aligning the left ventricular myocardium but not on other regions. Generally these effects reduce the imaged arterial activity and vary greatly among patients, indicating the need for individualized anatomic selection instead of using a fixed, "one-size-fits-none" region of interest. Recovering less than the full arterial input leads to overestimated and hence biased flow.

Results from a flow model must associate with clinical outcomes (as modified by medical treatment versus revascularization) in a large cohort followed for years, displaying a risk continuum with both size and severity. ${ }^{14}$ Incorporating flow quantification into clinical care and imaging reports should become the daily routine, as it has been at our center since April 2007 now over 14 years and exceeding 9,200 sequential PET scans. Its application in practice should be demonstrated to be cost-effective $\mathrm{e}^{15}$ and validated through randomized clinical trials. ${ }^{16}$

We have systematically examined each of these items (animal validation, flow spectrum from normal to ischemia, several pharmacologic stress medications, 
test/retest variability, arterial input customization, clinical outcomes, economic analysis, and randomized trial) in a series of publications mainly over the past decade, ${ }^{6-12,14-16}$ indicating that judging a PET flow model requires an edifice that cannot be built overnight and does not rest solely on any single aspect. Rather, multifaceted and interwoven evidence supports customized decisions for each patient based on the sound integration of PET flow physiology with unique clinical circumstances.

\section{SAME DATA, DIFFERENT RESULTS?}

With this background we can place the new results in context. ${ }^{5}$ In almost 50 patients with hypertrophic cardiomyopathy, dipyridamole stress N-13 ammonia PET images were obtained on a single scanner at one center in Italy. Reconstructed images were anonymized and shared among three centers (Italy, Finland, and Switzerland), each using a different software package (PMOD, Carimas, and FlowQuant, respectively) but the same one-tissue compartment model. After blinded analysis, global, territory, and segmental stress perfusion were compared among the three tools. Segmental values could vary by as much as $36 \%$ as quantified by the maximum/minimum ratio, and pairwise comparison of segments showed several outside of the their "acceptable agreement" range encompassing $\pm 20 \%$ in median value and $\geq 0.75$ intraclass correlation coefficient.

Because the reconstructed PET data and timeactivity curve model remained constant, the observed variation in the results can only have arisen due to model implementation or to differences in the input to the model (time-activity curves for the arterial input and myocardium uptake) or to a combination of both factors. The authors did not analyze the same time-activity curve in all three software packages, which would have uncovered any variations in implementation. Therefore, their results remain somewhat ambiguous regarding the mechanism(s) for the observed heterogeneity.

Nevertheless, that such frequent and sizable variations in calculated flow could arise from the same underlying data emphasizes the lack of portability among software packages. Consequently, in our opinion each vendor must be judged according to the integrative framework of Table 1. As described earlier, we believe that differences in the arterial input location ${ }^{12}$ likely account for a sizable portion of the variation in the flows observed in the current study. ${ }^{5}$ Additionally, many algorithms average over a region of interest in the myocardium to determine its uptake, which neglects basic physical principles of the point spread function and partial volume effects. ${ }^{17}$ To our knowledge, the partial volume factor remains "hidden in the black box", for almost all software vendors, providing a fudge factor never displayed to the user and hence not comparable among results.

Unexpectedly, the current analysis found the largest variation in quantification of stress perfusion in the lateral territory and segments. ${ }^{5}$ In normal volunteers the lateral wall has been shown by $\mathrm{PET}^{7}$ and invasive assessment $^{18}$ to have equivalent perfusion to the other regions. The discrepant results in this new manuscript did not arise due to a perfusion defect, since the lateral territory and segments had the highest average stress perfusion. ${ }^{5}$ Because each software package started with the same input image, this finding suggests spatial heterogeneity when generating time-activity curves for the left ventricle.

\section{FLOW PACKAGE, NOT FLOW MODEL}

In our view, the current manuscript by Nesterov et $\mathrm{al}^{5}$ offers a profound yet unexpected insight: flow software must be judged by more than its underlying physiologic model. Indeed, we should instead speak of a PET flow "package", that explicitly acknowledges many design choices that affect the numeric values: time-activity curve versus retention flow model, peak versus average myocardial uptake, fixed and known versus variable and hidden partial volume correction, and customized versus static arterial input location. Additionally, each flow package should be rated using the report card in Table 1. We do not think of people as interchangeable, although we are all based on the same "model" - the same holds true for PET flow software.

\section{Disclosures}

NPJ and KLG received internal funding from the Weatherhead PET Center for Preventing and Reversing Atherosclerosis; and have pending patents on diagnostic methods for quantifying aortic stenosis and TAVI physiology, and on algorithms to correct pressure tracings from fluid-filled catheters. NPJ has received significant institutional research support from St. Jude Medical (CONTRAST, NCT02184117) and Philips/Volcano Corporation (DEFINE-FLOW, NCT02328820) for other studies using intracoronary pressure and flow sensors; and has an institutional licensing agreement with Boston Scientific for the smart minimum FFR algorithm commercialized under 510(k) K191008. KLG is the 510(k) applicant for CFR Quant (K113754) and HeartSee (K143664, K171303, and K202679), software packages for cardiac positron emission tomography image processing, analysis, and absolute flow quantification. 


\section{References}

1. Bing RJ, Hammond MM, Handelsman JC, Powers SR, Spencer FC, Eckenhoff JE. The measurement of coronary blood flow, oxygen consumption, and efficiency of the left ventricle in man. Am Heart J 1949;38:1-24.

2. Gould KL, Schelbert HR, Phelps ME, Hoffman EJ. Noninvasive assessment of coronary stenoses with myocardial perfusion imaging during pharmacologic coronary vasodilatation. $\mathrm{V}$. Detection of 47 percent diameter coronary stenosis with intravenous nitrogen-13 ammonia and emission-computed tomography in intact dogs. Am J Cardiol 1979;43:200-208.

3. Schelbert HR, Wisenberg G, Phelps ME, Gould KL, Henze E, Hoffman EJ, et al. Noninvasive assessment of coronary stenoses by myocardial imaging during pharmacologic coronary vasodilation. VI. Detection of coronary artery disease in human beings with intravenous N-13 ammonia and positron computed tomography. Am J Cardiol 1982;49:1197-207.

4. Murthy VL, Bateman TM, Beanlands RS, Berman DS, et al. Clinical quantification of myocardial blood flow using PET: Joint position paper of the SNMMI Cardiovascular Council and the ASNC. J Nucl Cardiol 2018;25:269-97.

5. Nesterov SV, Sciagrà R, Orozco LEJ, Prior JO, et al. One-tissue compartment model for myocardial perfusion quantification with N-13 ammonia PET provides matching results: A cross-comparison between Carimas, FlowQuant, and PMOD. J Nucl Cardiol 2021. https://doi.org/10.1007/s12350-021-02741-4.

6. Yoshida K, Mullani N, Gould KL. Coronary flow and flow reserve by PET simplified for clinical applications using rubidium- 82 or nitrogen-13-ammonia. J Nucl Med 1996;37:1701-12.

7. Sdringola S, Johnson NP, Kirkeeide RL, Cid E, Gould KL. Impact of unexpected factors on quantitative myocardial perfusion and coronary flow reserve in young, asymptomatic volunteers. JACC Cardiovasc Imaging 2011;4:402-12.

8. Johnson NP, Gould KL. Physiological basis for angina and STsegment change PET-verified thresholds of quantitative stress myocardial perfusion and coronary flow reserve. JACC Cardiovasc Imaging 2011;4:990-8.

9. Kitkungvan D, Johnson NP, Roby AE, Patel MB, Kirkeeide R, Gould KL. Routine clinical quantitative rest stress myocardial perfusion for managing coronary artery disease: Clinical relevance of test-retest variability. JACC Cardiovasc Imaging 2017;10:56577.

10. Kitkungvan D, Lai D, Zhu H, Roby AE, Johnson NP, Steptoe DD, et al. Optimal adenosine stress for maximum stress perfusion, coronary flow reserve, and pixel distribution of coronary flow capacity by Kolmogorov-Smirnov analysis. Circ Cardiovase Imaging 2017;10:e005650.

11. Johnson NP, Gould KL. Regadenoson versus dipyridamole hyperemia for cardiac PET imaging. JACC Cardiovasc Imaging 2015;8:438-47.

12. Vasquez AF, Johnson NP, Gould KL. Variation in quantitative myocardial perfusion due to arterial input selection. JACC Cardiovasc Imaging 2013;6:559-68.

13. Franks R, Milidonis X, Schneider T, Sánchez-González J, Plein S, Chiribiri A. Impact of the arterial input sampling location on CMR first-pass myocardial perfusion quantification. JACC Cardiovasc Imaging 2020;13:2693-5.

14. Gould KL, Kitkungvan D, Johnson NP, Nguyen T, Kirkeeide R, Bui L, et al. Mortality prediction by quantitative PET perfusion expressed as coronary flow capacity with and without revascularization. JACC Cardiovasc Imaging 2021;14:1020-34.

15. Delgado RI, Swint JM, Lairson DR, Johnson NP, Gould KL, Sdringola S. Cost analysis of PET and comprehensive lifestyle modification for the reversal of atherosclerosis. J Nucl Med 2014;55:80-7.

16. Kitkungvan D, Johnson NP, Kirkeeide R, Haynie M, Carter C, Patel MB, et al. Design and rationale of the randomized trial of comprehensive lifestyle modification, optimal pharmacological treatment and utilizing PET imaging for quantifying and managing stable coronary artery disease (the CENTURY study). Am Heart J 2021;237:135-46.

17. Johnson NP, Sdringola S, Gould KL. Partial volume correction incorporating $\mathrm{Rb}-82$ positron range for quantitative myocardial perfusion PET based on systolic-diastolic activity ratios and phantom measurements. J Nucl Cardiol 2011;18:247-58.

18. Fournier S, Keulards DCJ, van 't Veer M, Colaiori I, et al. Normal values of thermodilution-derived absolute coronary blood flow and microvascular resistance in humans. EuroIntervention 2021;17:e309-16.

Publisher's Note Springer Nature remains neutral with regard to jurisdictional claims in published maps and institutional affiliations. 